\title{
Conocimiento de la enseñanza de números racionales: una ejemplificación de relaciones
}

\author{
Conhecimento do ensino de números racionais: uma exemplificação de relações
}

\section{Knowledge of mathematics teaching rational numbers: an example of relationships}

\author{
Diana Zakaryan ${ }^{1}$ \\ Miguel Ribeiro ${ }^{2}$
}

\begin{abstract}
Resumen
El objetivo de este artículo es evidenciar y caracterizar las relaciones entre distintos subdominios del conocimiento matemático especializado del profesor de matemáticas. Particularmente, buscando la mejora de la práctica del profesor, estas relaciones y su rol en la práctica tienen relevancia en las situaciones matemáticamente críticas, una de las cuales se refiere a la enseñanza y el aprendizaje de los números racionales. Considerando el conocimiento del profesor desde el modelo Mathematics Teachers Specialized Knowledge, se identifican y se caracterizan las relaciones entre uno de los subdominios (Knowledge of Mathematics Teaching) y los demás subdominios del conocimiento del profesor de matemáticas, en la práctica de una profesora chilena ( $8^{\circ}$ grado - alumnos de 13-14 años), en el tema de los números racionales. Se concluye presentando estas relaciones mediante mapas de conexiones entre los subdominios del modelo.
\end{abstract}

Palavras-chave: Mathematics Teachers Specialized Knowledge; conocimiento didáctico del contenido; conocimiento de la enseñanza de las matemáticas; números racionales.

\section{Resumo}

Este artigo tem por objetivo evidenciar e caracterizar as relações entre distintos subdomínios do conhecimento matemático especializado do professor que ensina matemática. Em particular, buscando uma melhora da prática do professor, estas relações e o seu papel na prática assumem uma relevância central nas situações matematicamente criticas, tal como seja o ensino e aprendizagem dos números racionais. Considerando o conhecimento do professor segundo o Mathematics Teachers Specialzid Knowledge, identificamos e caracterizamos as relações entre um dos subdomínios (Knowledge of Mathematics Teaching) e os demais subdomínios do conhecimento do professor a partir da análise da prática de uma professora chilena (Anos Finais do Ensino Fundamental) no tema dos racionais. Concluímos apresentando as relações entre os vários

\footnotetext{
${ }^{1}$ Doctora en Didáctica de la Matemática por la Universidad de Huelva (UHU), España. Profesora del Instituto de Matemáticas, Pontificia Universidad Católica de Valparaíso, Chile. E-mail: diana.zakaryan@pucv.cl.

2 Doctor en Didáctica de la Matemática por la Universidad de Huelva (UHU), España. Profesor de la Universidade Estadual de Campinas - UNICAMP, Brasil. E-mail: cmribas78@gmail.com.
} 
subdomínios do conhecimento do professor que ensina matemática por meio de um mapa de conexões.

Keywords: Mathematics Teachers Specialized Knowledge; conhecimento pedagógico do conteúdo; conhecimento do ensino de matemática; números racionais.

\begin{abstract}
This paper aims at characterizing the relationships between different subdomains of mathematics teachers' specialized knowledge. In particular, when searching for improving teachers' practices, such relationships and its role in practice have a crucial role in the mathematical critical situations, such as the teaching and learning of rational numbers. Considering teachers' knowledge in the perspective of the Mathematics Teachers Specialized Knowledge, an identification and characterization of the relationships between one of its subdomains (Knowledge of Mathematics Teaching) and the remaining is made. For doing so, the practice of a Chilean upper secondary teacher is analysed, when she is teaching rational numbers. Using a map of connections, a set of relationships between different subdomains is presented. Some future pathways for research are presented.
\end{abstract}

Keywords: Mathematics Teachers Specialized Knowledge; pedagogical content knowledge; knowledge of mathematics teaching; rational numbers.

\title{
Introducción
}

Cuando se analiza el currículo oficial de distintos países, se observa que los números racionales, fracciones en primaria, es uno de los contenidos que aparece de manera transversal en todas las etapas educativas (e.g., Ministerio de Educación de Chile, 2009). Esa transversalidad de los racionales, concretamente en primaria, está justificada por el hecho de que los números racionales son uno de los contenidos matemáticos más complejos e importantes en los niveles iniciales (e.g., Behr, Lesh, Post \& Silver, 1983; Lamon, 2007; Kieren, 1976) dado que la génesis de su plena comprensión se encuentra en el desarrollo de estructuras cognitivas cruciales para el aprendizaje de tópicos matemáticos en niveles más avanzados. Las dificultades de los alumnos con los números racionales se configuran como un obstáculo en el desarrollo de su conocimiento matemático, que puede tener su origen en la utilización precoz de reglas y algoritmos en el estudio de los racionales (Behr et al., 1983); o en la falta de experiencias concretas, necesarias para la construcción de la comprensión conceptual de la fracción; o en la falta de conexiones entre esas experiencias y los conceptos abstractos debido a un desarrollo débil del razonamiento multiplicativo (Vanhille \& Baroody, 2002). De hecho, la comprensión de muchos de los conceptos asociados a los racionales se asocian a la estructura multiplicativa entre números enteros (e.g., Flores, 2002; Vergnaud, 1983).

Por otro lado, considerando el papel del profesor y de su conocimiento en los aprendizajes de los alumnos (e.g., Nye, Konstantopoulos \& Hedges, 2004), dichas dificultades pueden sustentarse en dificultades similares de los profesores (e.g., Graeber, Tirosh \& Glover, 1989; Harel, Behr, Post \& Lesh, 1994; Ma, 1999) ya que estos se enfrentan con el mismo tipo de dificultades identificadas en los alumnos (e.g., Jakobsen, Ribeiro \& Mellone, 2014; Pinto \& Ribeiro, 2013). En este sentido, con el fin de solventar las 
dificultades de los alumnos, particularmente, en los racionales, se torna esencial que los profesores tengan conocimiento de y sobre el tema que les permita preparar e implementar tareas matemáticamente desafiantes (e.g., Jakobsen et al., 2014; Stein, Smith, Henningsen \& Silver, 2000). Dado que el concepto de los números racionales, tal como mencionamos anteriormente, es un tema que presenta dificultades en su comprensión tanto para alumnos como para profesores y, por otra parte, faltan estudios enfocados en la práctica del profesor en el tema a nivel de la enseñanza secundaria (e.g., discutiendo racionales en un sentido más profundo del que se enfocan en primaria), el foco de interés de este estudio es el conocimiento del profesor de los números racionales en el nivel de la enseñanza secundaria.

Al referirse al conocimiento del profesor se encuentra una variedad de perspectivas para su estudio. La mayoría de dichas perspectivas se basan en los trabajos de Shulman (1986) y en la categorización de los dos subdominios del conocimiento del profesor: Conocimiento del Contenido (SMK) y Conocimiento Pedagógico del Contenido (PCK). Desde esa aportación de Shulman (1986), surgieron varias conceptualizaciones del conocimiento del profesor de matemáticas que consideran en los dos dominios algunas especificaciones de manera que permiten su operacionalización a la hora de identificar el contenido de cada uno de ellos (e.g., Mathematical Knowledge for Teaching - Ball, Thames \& Phelps, 2008; Mathematics Teacher Specialized Knowledge - MTSK - Carrillo, Climent, Contreras \& Muñoz-Catalán, 2013). En estas propuestas se destaca la idea de la existencia de conocimientos de distinta índole, interrelacionados y suficientes para proporcionar una práctica docente integrada y generadora de diversas y ricas oportunidades de aprendizaje para los alumnos. En este documento, el conocimiento del profesor de matemáticas se trata desde la perspetiva del MTSK, considerando exclusivamente aquellos conocimientos del profesor que tengan relación directa con el contenido matemático. De esta manera, no se consideran como parte del conocimiento especializado, por ejemplo, el conocimiento del profesor de técnicas de control del grupo o de trabajo en equipo (e.g., Flores-Medrano, Escudero-Avila, Montes, Aguilar \& Carrillo, 2014).

La preocupación y deseo de contribuir de forma activa y participativa a la mejora de la práctica docente y a la formación de profesores nos lleva a la búsqueda de la comprensión de ese complejo y multidimensional constructo del conocimiento del profesor, permitiendo (i) caracterizar el conocimiento de la enseñanza de las matemáticas (KMT), enfocándose en la práctica docente en el aula de una profesora y (ii) evidenciar relaciones entre el KMT y otros subdominios del MTSK. Proceder en esta caracterización y evidenciar las relaciones entre subdominios del MTSK, tiene por objetivo vislumbrar la complejidad de cada uno de los subdominios del conocimiento del profesor y la diversidad de relaciones entre estos, así como, de manera asociada, contribuir con elementos para su desarrollo. Asimismo, ejemplificar el uso del modelo MTSK para el análisis del conocimiento del profesor a partir de su práctica docente, puede ser de utilidad como una herramienta analítica a los futuros 
profesores y profesores en ejercicio para darse cuenta y reflexionar sobre su propio conocimiento, así como a sus formadores, revelando elementos para una proyección oportuna de los aprendizajes de sus estudiantes.

\section{Marco teórico}

El conocimiento del profesor tiene una influencia directa en su práctica del aula según los objetivos que persiga (e.g., Nye et al., 2004; Ribeiro \& Carrillo, 2011), el conocimiento que tiene de los aprendizajes de los alumnos y de sus dificultades (Zakaryan, Ribeiro \& Carrillo, sometido), entre otros. En este sentido, con el fin de mejorar, en última instancia los aprendizajes de los alumnos, y dado que el conocimiento didáctico del contenido (el que permite, en primera instancia, al alumno interactuar con los contenidos matemáticos) se sustenta en el conocimiento del contenido (e.g., Ma, 1999), se torna esencial una discusión acerca del conocimiento didáctico del contenido de los números racionales y el desarrollo del conocimiento del profesor en este contenido.

Entre los distintos modelos/conceptualizaciones del conocimiento del profesor que se puede encontrar en la literatura científica, este trabajo se desarrolla bajo la conceptualización del modelo MTSK, presentada en la Figura 1 (Muñoz-Catalán, Contreras, Carrillo, Rojas, Montes, \& Climent, 2015). Este modelo atañe la parte del conocimiento profesional del profesor de matemáticas que está condicionada por la propia matemática, es decir, no incluye otros dominios del modelo de Shulman (1986), también pertenecientes al conocimiento profesional como, por ejemplo, el conocimiento psicopedagógico general.

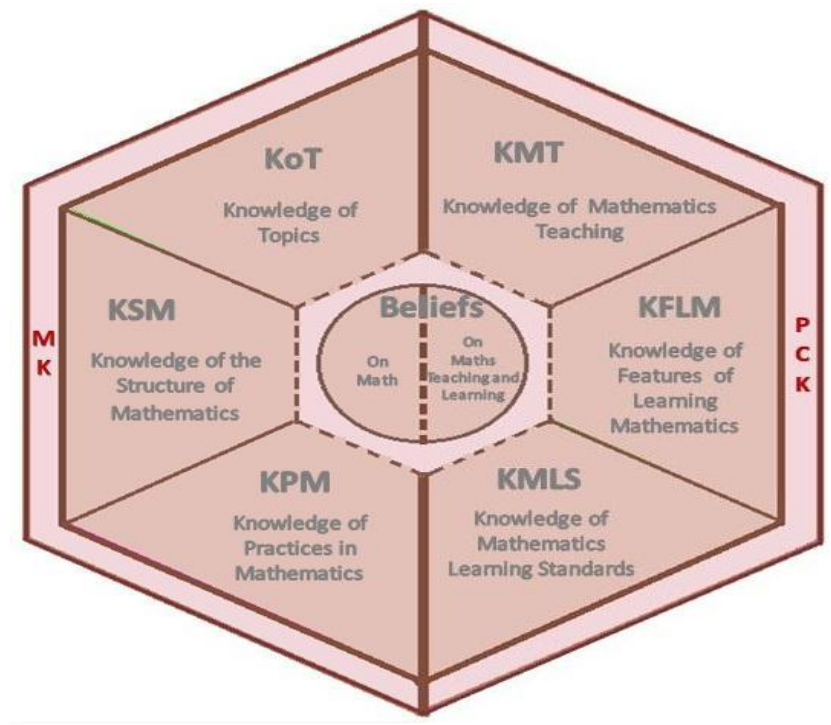

Figura 1. Mathematics Teacher's Specialized Knowledge Fuente: Muñoz-Catalán et al., 2015 
En el MTSK se consideran dos grandes dominios: Conocimiento Matemático (MK) y Conocimiento Didáctico del Contenido (PCK), y seis subdominios de naturaleza diferenciable, tres por cada dominio. El MTSK considera, asimismo, un dominio de creencias/concepciones del profesor sobre la matemática y sobre su enseñanza y aprendizaje, el cual está íntimamente ligado a los subdominios anteriores - de asumiendo el centro del modelo del conocimiento del profesor.

Dado que el foco de este artículo es identificar y evidenciar las relaciones existentes entre los contenidos del subdominio Conocimiento de la Enseñanza de las Matemáticas (KMT) y los contenidos de otros subdominios del MTSK, presentamos brevemente los subdominios del MTSK, haciendo una discusión más amplia y detallada del subdominio KMT.

Conocimiento de los Temas (KoT): este subdominio contempla más que el conocimiento de la matemática como disciplina, incluyendo la matemática escolar y más allá de lo que sus alumnos aprenden, así como lo relativo a sus fundamentos matemáticos, los procedimientos, estándares y alternativos, o las distintas formas de representación de los diferentes temas. Este subdominio incluye aspectos de los conceptos que permiten relacionarlos con contextos reales o con el propio contenido matemático en forma de ejemplos, aportando aspectos epistemológicos ligados a la matemática que permiten al profesor comprender diferentes significados que pueden atribuirse al contenido, así como una amplia variedad de contextos en los que situarlo. Por ejemplo, saber la fenomenología de los números racionales, su definición (o incluso distintas definiciones, dependiendo del punto de partida o del abordaje histórico).

Conocimiento de la Estructura de la Matemática (KSM): este subdominio abarca el conocimiento de las matemáticas desde la perspectiva de su integración y relación en estructuras amplias y con mayor capacidad de relación con otros conceptos que lo «local» de un concepto determinado. Este subdominio integra tanto aquellas relaciones con conceptos más avanzados, como más elementales, que permiten al profesor trabajar la matemática avanzada desde un punto de vista elemental y viceversa. Asimismo, las grandes ideas constituyen un elemento estructurador de la matemática, generando conexiones de tipo transversal. Un ejemplo de este tipo de conexión sería el conocimiento del profesor de la estructura de los distintos sistemas numéricos (e.g., naturales, enteros, racionales, reales) y conexiones entre dichos conjuntos $(\mathbf{N} \subset \mathbf{Z} \subset \mathbf{Q} \subset \mathbf{R})$.

Conocimiento de la Práctica Matemática (KPM): este subdominio abarca aquellas formas de hacer y proceder en matemáticas que un profesor ha de conocer para desarrollar su clase, como son las diferentes formas de demostrar, los criterios para establecer una generalización válida, el significado de definición, axioma o teorema como elementos constituyentes de la matemática, o el conocimiento de la sintaxis matemática. Asimismo, 
tiene en este subdominio un papel relevante el conocimiento de distintos heurísticos en resolución de problemas, que abarcan la estructura lógica en la que se desarrolla la resolución. Saber que una manera de proceder en matemáticas es buscar y crear patrones, sería un ejemplo de este subdominio.

Por otra parte, el Conocimiento Didáctico del Contenido considera los siguientes tres subdominios:

Conocimiento de las Características del Aprendizaje de las Matemáticas (KFLM): este subdominio refleja el conocimiento que el profesor posee acerca de cómo se aprenden y piensan los contenidos matemáticos, así como de las formas que tienen los alumnos de interactuar con cada contenido. Este subdominio incluye el conocimiento de diferentes teorías, personales o institucionalizadas, de aprendizaje de las matemáticas, el conocimiento de las fortalezas, dificultades, obstáculos, o errores, asociados a cada contenido, así como el lenguaje o vocabulario habitualmente usado por los estudiantes en cada contenido. También podemos encontrar aquí el conocimiento del profesor de las ideas intuitivas habitualmente desarrolladas por los alumnos al tratar con ciertos conceptos, así como el conocimiento de aspectos propios ligados a las actitudes hacia la matemática. Por ejemplo, el conocimiento acerca de las dificultades que presentan los alumnos para ordenar los números racionales, o un profesor puede conocer que los conocimientos anteriores de los alumnos de las propiedades y operaciones con números naturales, hacen que estos tiendan a transferirlas, de forma directa, a las operaciones con fracciones.

Conocimiento de los Estándares de Aprendizaje de las Matemáticas (KMLS): este subdominio abarca los diferentes grados de profundidad en que un profesor pudiera conocer el currículo oficial, respecto de las matemáticas, vigente en el país en que imparte su docencia, y su concreción, en caso de existir, en un territorio concreto del mismo. Asimismo, incluye el conocimiento de los contenidos y capacidades que debe aprenderse y desarrollarse en un curso o etapa, con indicaciones de la forma en que deben impartirse y aprenderse los contenidos. Este tipo de referentes existen fuera del currículo, pudiendo un profesor conocer lo que ciertas asociaciones profesionales como la National Council of Teachers of Mathematics (NCTM) proponen en ese sentido, o también la opinión de profesores expertos, con gran experiencia y conocimiento de la práctica docente sobre qué, cómo y cuándo explicar los contenidos matemáticos. Por ejemplo, el conocimiento del profesor de que el número racional (fracción) se trabaja en la educación básica usando principalmente la representación gráfica, cabría en este subdominio.

El Conocimiento de la Enseñanza de las Matemáticas (KMT), que se configura como el foco de interés de este estudio, considera solo aquellos conocimiento relacionados con la enseñanza de las matemáticas donde el contenido matemático condiciona la enseñanza y no incluye, por tanto, conocimientos pedagógicos en contextos de actividades matemáticas 
(Escudero-Avila, 2015). El KMT incluye el conocimiento que tiene el profesor de las vías, recursos y formas de enseñar matemáticas, de diferentes estrategias y teorías, institucionalizadas o personales de enseñanza de las matemáticas.

Asimismo, es especialmente relevante aquí el conocimiento de diferentes recursos para la enseñanza de las matemáticas, como pueden ser el dominó de números racionales, en relación con su potencial y limitaciones para la enseñanza de las fracciones equivalentes. Cabe destacar aquí que el conocimiento de diferentes representaciones en sí de un objeto matemático no se considera en este modelo como conocimiento didáctico, como lo suele considerar otros autores (e.g., Shulman, 1986). Por ejemplo, los diferentes tipos de representación de los números racionales no implican conocer dificultades en el aprendizaje de las operaciones con ellos que dependen directamente del tipo de representación usada, ni conocer estrategias específicas de uso de una determinada representación. Conocer dichas representaciones es más bien de índole matemático y está considerado en el Conocimiento de los Temas (KoT), en la categoría de registros de representación. El conocimiento didáctico de dicho contenido es el que tiene el profesor sobre las características matemáticas que hacen de esta representación la más propicia para utilizarla en la enseñanza, el cual necesariamente está ligado al dominio matemático, y a los demás subdominios del PCK, pero es, en sí misma, una forma distinta de conocer el contenido matemático (Escudero-Avila, 2015).

En el KMT se consideran tres categorías que se relacionan con las (i) teorías de enseñanza de las matemáticas; (ii) recursos materiales y virtuales e (iii) estrategias, técnicas y tareas asociadas a cada uno de los contenidos matemáticos.

(i) Teorías de enseñanza de las matemáticas: conocer teorías de enseñanza (institucionalizadas o personales) por ejemplo, un profesor puede conocer la teoría del Espacio de Trabajo Matemático (Kuzniak, 2011) y de ahí tratar de crear un ETM idóneo, eligiendo y organizando tareas de un contenido matemático concreto, de manera que se generen ETMs personales eficientes de los estudiantes.

(ii) Recursos materiales y virtuales: conocer características matemáticas específicas de recursos didácticos para la enseñanza de un determinado contenido matemático (e.g., el dominó de números racionales).

(iii) Estrategias, técnicas y tareas asociadas a un contenido matemático: conocer la potencialidad y las limitaciones de las estrategias y técnicas didácticas para enseñar un contenido concreto, el tipo de tareas apropiadas para el dicho contenido. Asimismo, los ejemplos elegidos para representar un contenido, las metáforas, las situaciones y las explicaciones potentes, constituyen conocimiento sobre la enseñanza del contenido.

Por ejemplo, el conocimiento del profesor sobre el hecho de que al tratar el significado de las fracciones solo como parte-todo, limitará su comprensión por parte de los alumnos, generando obstáculo didáctico, y por tanto, saber proponer ejemplos, tareas y tratar 
otros significados de la fracción para su entendimiento más completo, se incluiría en esta categoría. En este ejemplo podemos ver la relación entre el KMT, el KFLM y el KoT del profesor, dado que la elección de ejemplos, tareas y significados que se dará a la fracción generarán una cierta forma de aprendizaje de la fracción, además de tener conocimiento sobre las dificultades de los alumnos (KFLM) y conocer los diferentes significados de la fracción (KoT).

\section{Contexto y método}

Este documento tiene una doble finalidad: por una parte, caracterizar el conocimiento de la enseñanza de las matemáticas (KMT) de la profesora buscando relaciones con otros subdominios del modelo MTSK; por otra parte, ejemplificar cómo se puede usar el modelo MTSK para el análisis del conocimiento del profesor a partir de su práctica docente.

Desde un estudio de enfoque interpretativo se realiza un estudio de caso instrumental (Stake, 2007). El caso es una profesora (en adelante Ana) del $8^{\circ}$ grado de la educación básica chilena (alumnos de 13-14 años) que en el momento del estudio contaba con siete años de experiencia y es titulada como Profesora de Matemáticas y Licenciada en Educación.

La obtención de los datos se ha realizado a través de observaciones no participante de aula (Cohen \& Manion, 2002) mientras Ana imparte la unidad relacionada con los números racionales (7 sesiones), complementadas con grabaciones en video. Las videograbaciones han sido transcritas y a partir de dicha transcripción se ha hecho la modelización de la práctica de Ana recurriendo a una adaptación del modelo propuesto por Ribeiro et al. (2012) - Ilustración 1. En el proceso de transcripción, las sesiones se dividen en episodios (fenomenológicamente coherentes) en función de los objetivos matemáticos de la profesora. En cada uno de dichos episodios se identifican los subdominios del conocimiento evidenciado por la profesora, y se registran las evidencias de dicho conocimiento (transcripciones de la sesión). En el proceso de modelización los objetivos matemáticos del profesor juegan un papel central, permitiendo hacer la división de las sesiones en episodios de una manera coherente (e.g., la indicación [i,j] corresponde al episodio j de la sesión i). Cada uno de estos episodios viene delimitado por un evento inicial y un evento final.

\section{[i. j] Descripción del episodio [línea de inicio - línea de fin]}

Objetivo general: Identificación del objetivo del contenido matemático que pretende enseñar el profesor.

Evento desencadenante: Evento que funciona como causa de inicio del episodio.

Conocimientos (MTSK): Identificación de las evidencias de los subdominios del MTSK en la práctica de la profesora en ese periodo temporal - delimitado por las líneas de la transcripción.

Evento de término: Evento que funciona como causa de término de ese episodio.

$$
\text { Ilustración 1. Representación del instrumento de modelización de la práctica. }
$$


Una vez realizada la modelización de las sesiones y registrados los indicadores del MTSK, se procede en identificar y profundizar en la comprensión de las relaciones entre el conocimiento de la enseñanza de las matemáticas (KMT) con los demás subdominios del MTSK - de entre los que se observa en su actuación.

\section{Análisis de datos}

A modo de ejemplo, se presenta la modelización de un episodio y un extracto en el cual se registran los indicadores del MTSK y se identifican relaciones entre el KMT y otros subdominios del MTSK.

La primera sesión se divide en ocho episodios según los objetivos matemáticos de la profesora. El tercer episodio de la primera sesión [1.3], que se ejemplifica a continuación (Ilustración 2), tuvo por objetivo explorar la equivalencia entre números racionales con recurso el dominó. Este episodio, donde la profesora comunica a los alumnos que van a hacer las fichas y luego van a jugar dominó de números racionales y explica qué conocimientos van a necesitar para ello, está delimitado por un evento inicial (el comentario de la profesora para introducir el recurso material-dominó de números racionales, línea 123) y un evento final (término del comentario para seguir con la definición formal de los números racionales, 141).

\section{[1. 3] Juego del dominó de números racionales [123 - 141]}

Objetivo general: Exploración de la equivalencia de los números racionales.

Evento desencadenante: Comentario de Ana para introducir el recurso material- dominó de los números racionales.

KoT: Procedimientos (operar) con los números racionales; propiedades de los números racionales.

KMT: Conocimiento de recurso material-dominó de números racionales.

KFLM: Conocimiento de las expectativas e intereses de los alumnos respecto al aprendizaje de las matemáticas.

Evento de término: Ana termina su comentario.

$$
\text { Ilustración 2. Modelización del tercer episodio de la primera sesión. }
$$

En este extracto se evidencia el conocimiento de Ana de la enseñanza de las matemáticas (KMT), el conocimiento de las características del aprendizaje de las matemáticas (KFLM) y el conocimiento de procedimientos y propiedades (KoT), lo que corresponde a las líneas [123-130] de la transcripción (se destacan en negrita las evidencias del conocimiento que se van a considerar). 
[123] Ana: Vamos a hacer un trabajo que hicimos el año pasado con un curso, que

[124] resultó bien entretenido al final, es un dominó de números racionales.

[125] De eso se trata, aprendérselos de una forma más entretenida.

[126] Entonces, lo para poder jugar: Son equivalencias, poder saber que a

[127] lo mejor el 1 lo que usted tiene que saber escribe de esa forma $\left(\frac{3}{3}\right)$

[128] que el -5 se puede escribir de esa forma $\left(\frac{-5}{1}\right)$. Cuando uno va jugando

[129] al dominó, el compañero puede tirar el -5, y el otro tiene esta fracción,

[130] entonces juega con esa [...]

Ilustración 3. Extracto de transcripción correspondiente al episodio [1.3], donde la profesora tiene por objetivo explorar la equivalencia de números racionales.

El KMT de Ana, en este extracto, se refiere al conocimiento de recurso material para el tratamiento de la equivalencia de los números racionales, del dominó de números racionales, líneas [123-130]. Por otra parte, se observan evidencias de su conocimiento de expectativas e intereses de los alumnos al/para aprender matemáticas (KFLM), líneas [123125] y su conocimiento de propiedades y procedimientos con los números racionales (KoT), líneas [126-130].

La identificación de los subdominios del MTSK revelados por la profesora en la práctica, permite también identificar/discutir la existencia (o no) de relaciones entre distintos subdominios considerando, en particular, el KMT como punto de partida. Así, enfocando nuestra atención en los episodios donde hay evidencias del KMT, se elaboran mapas de conexiones entre las categorías de ese subdominio del PCK y las de los demás subdominios identificados. El mapa de conexiones para el extracto presentado anteriormente quedaría de la siguiente manera (Figura 2):

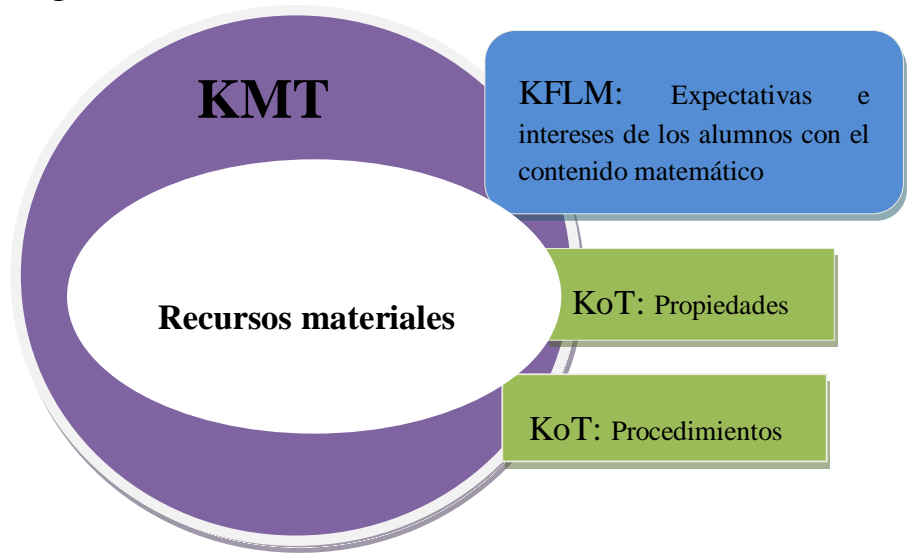

Figura 2. Mapa de conexiones entre el KMT y otros subdominios del MTSK.

Fuente: Elaboración propia. 
De este modo, el KMT de Ana (recursos materiales) está relacionado con su conocimiento de intereses de los alumnos al aprender matemáticas (KFLM). Ana menciona, explícitamente durante la clase, que "con ese juego los alumnos van a aprender de una manera entretenida". Aunque el foco de Ana aquí es el recurso, y la intención de desarrollar la práctica de una "manera entretenida", la elección de dicho recurso se sustenta en su conocimiento matemático de que todo número racional puede escribirse como una familia infinita de fracciones equivalentes (propiedades de los números racionales-KoT), así como buscar y reconocer equivalencias (procedimientos con números racionales-KoT).

De manera similar se analizan las siete sesiones de clases de Ana y los resultados de este análisis se presentan en el siguiente apartado.

\section{Relaciones entre el KMT y otros subdominios del MTSK}

En este apartado se presentan los indicadores evidenciados del conocimiento de la enseñanza de las matemáticas de la profesora y sus relaciones con otros subdominios del modelo MTSK.

En el siguiente extracto (Ilustración 4) la profesora solicita a los alumnos nombrar los números que conocen desde que vienen al colegio:

\begin{tabular}{|ll}
\hline$[10]$ Ana: & ¿Qué números conocen ustedes desde que empezaron a venir al colegio? \\
{$[11]$} & Ya, deme ejemplos de números. Pero no me los nombre todos de aquí al \\
{$[12]$} & infinito... \\
$\ldots$ & $\ldots$ \\
{$[49]$} & Ya, entonces nos vamos a quedar con algunos ejemplos, no con todos. \\
{$[50]$} & Usted coloque ahí: Ejemplos, puede ser el 0; el 1; el -5; el -0,5;...-1,3.
\end{tabular}

Ilustración 4. Extracto de transcripción del episodio [1.1], donde el objetivo de la profesora es introducir el conjunto de los números racionales.

En este extracto se observa que Ana hace alusión a que existen infinitos números, cuando dice que no le nombren todos los números, a continuación, siguiendo la misma idea, destaca que se quedarán con algunos ejemplos de los infinitos que pueden existir. El hecho de conocer ejemplos conforma el KoT de la profesora, mientras proponer ejemplos en esta situación particular, se asociaría con el KMT de la profesora. Por otra parte, Ana manifiesta conocer conexiones entre el tratamiento adecuado para dicho nivel del concepto de infinitud de los números de ese concepto matemático avanzado, desde una perspectiva elemental (KSM).

El siguiente fragmento del episodio, donde el objetivo de la profesora es identificar los elementos del conjunto de los números racionales, da evidencia del conocimiento de la enseñanza de los números racionales, particularmente, su conocimiento sobre el uso de 
distintas representaciones de los números racionales con el fin de potenciar la comprensión de los alumnos:

\begin{tabular}{|c|c|}
\hline [64] Alumno: & Profesora, ¿por qué uno es $2 / 2 ?$ \\
\hline [65] Ana: & Porque si uno se detiene en lo que acabamos de escribir, que dice que \\
\hline [66] & cualquier número se puede escribir como fracción. Esto es una \\
\hline [67] & fracción, pero a la vez una en 2 ¿Cuánto te da? 1, ahí está. Por lo \\
\hline [68] & tanto, al tener esto, es equivalente a tener $1 \ldots 1 / 2$, dividamos $1 / 2$ para \\
\hline [69] & salir de la duda. (Escribe $1: 2=$ ) ¿Podemos hacer de inmediato esta \\
\hline [70] & división? \\
\hline [71] Alumno: & No \\
\hline [72] Ana: & Entonces ¿Qué tenemos que hacer? \\
\hline [73] Alumno: & Cero... \\
\hline [74] Ana: & ¿Y luego? (alumnos realizan la división). Mire lo que acaba de dar \\
\hline [75] & $(0,5)$. Eso es al dividirlo, y si tú hubieses realizado otra \\
\hline [76] & representación, como lo hacen en básica, $1 / 2$ ¿Qué significa si yo \\
\hline [77] & hago un dibujito ahí? La mitad ¿cierto?, por lo tanto, no puedo \\
\hline [77] & pensar que ese $1 / 2$ es 1 , porque al dividirlo me da la mitad y al \\
\hline [78] & representarlo también me da la mitad, no me da el entero que estamos \\
\hline [79] & buscando. También podría ser $3 / 3,4 / 4$ y etc. \\
\hline
\end{tabular}

El KMT de la profesora está relacionado, por una parte, con su conocimiento de las potencialidades de temas anteriores para cursos posteriores a un determinado momento escolar (KMLS). Mencionando "como lo hacen en básica", Ana evidencia su conocimiento de cómo se representa el número racional en los niveles anteriores, aprovechando ese conocimiento previo de los alumnos y dando diferentes representaciones de un número racional (gráfica y numérica), entre ellos ya conocida por los alumnos, potenciando el entendimiento de los alumnos. Por otra parte, su KMT se sustenta en su conocimiento de diferentes representaciones de los números racionales (KoT).

En el siguiente extracto, Ana además de manifestar su conocimiento de que el hecho de que los alumnos no sepan leer correctamente la notación decimal (y, luego, asociado al desarrollo del sentido numérico) les va a dificultar el transformar un número decimal finito a fracción, que sería su KFLM, muestra saber cómo solventar esa dificultad de los alumnos, usando un lenguaje con significado asociado a la cantidad que se desea expresar (el número) y poniendo ejemplos (KMT). 


\begin{tabular}{|ll|}
\hline$[93]$ Ana: & $\ldots(0,2) \cdot \frac{2}{10}$, escuché por ahí. ¿Cómo asociar la fracción \\
{$[94]$} & inmediata de esos números? ¿Cómo se lee? Se supone que el 2 \\
{$[95]$} & ocupa la décima, por lo tanto, cómo se lee? ¿No se llama $\mathbf{0 , 2}$ \\
{$[96]$} & [cero coma dos]? Dos décimos. Así como lo nombro. Si usted \\
{$[97]$} & tuviera, por ejemplo, este otro 0,03.¿Cómo se lee eso? \\
{$[98]$ Alumnos: } & Tres centésimos. $3 / 100$ \\
{$[99]$ Ana: } & Suelen complicarse con saber cuál es la fracción asociada porque \\
{$[100]$} & no sabemos leer el número. \\
\hline \multicolumn{2}{|c|}{ Ilustración 6. Extracto de transcripción correspondientes al episodio [1.2], donde el objetivo de la } \\
profesora es identificar los elementos del conjunto de los números racionales.
\end{tabular}

Por otra parte, en este extracto se puede notar el KoT de la profesora, asociado al significado matemático de la expresión numérica.

Asimismo, más adelante, en el segundo episodio de la segunda sesión [2.2], donde el objetivo es transformar los decimales a fracciones, se puede observar que Ana usa una técnica de la enseñanza que consiste en buscar patrones para la transformación de decimales a fracción, a partir de los ejemplos que presenta:

\begin{tabular}{|ll|}
\hline$[347]$ Ana: & ..Vamos a partir por transformación de decimales a fracción, pero \\
{$[348]$} & vamos a partir por el primero que sería el finito. Deme un ejemplo \\
{$[349]$} & de un finito. El compañero dice "el 0,1". Ahí tenemos un finito. \\
{$[350]$} & ¿Cómo se lee ese número? \\
{$[351]$ Alumno: } & Un décimo. \\
{$[352]$ Ana: } & Ya, lo voy a anotar aquí, un décimo. Entonces, ¿cómo lo puedo \\
{$[353]$} & escribir como fracción? \\
{$[354]$ Alumno: } & 1 partido en 10 \\
$\ldots$ & $\ldots$ \\
{$[361]$ Ana: } & Y vamos a agregar el último (0,352) ¿cómo se lee ese? 352 \\
{$[362]$} & milésimas, y ¿cómo lo escribo como fracción? \\
{$[363]$ Alumno: } & 352 partido en 1000 \\
{$[364]$ Ana: } & Si no pasáramos por la lectura, haga cuenta que no lo hicimos, \\
{$[365]$} & icuál sería una regla para que esto pasara a fracción? \\
{$[366]$} & Vea las fracciones, ¿cuál sería la regla? \\
{$[367]$ Alumnos: } & Por la cantidad de decimales que hay, la cantidad de ceros. \\
{$[368]$ Ana: } & Entonces ¿cuál sería la regla? Pero si usted me dice eso, por \\
{$[369]$} & cada decimal, coloco un cero debajo... Le voy a dar otro ejemplo. \\
{$[370]$} & (2,37) por cada cifra decimal coloco un cero debajo, eso es lo que \\
{$[371]$} & usted me acaba de decir, pero eso no hace que sea una fracción \\
{$[372]$} & todavía, algo falta. Hay que hacer bien una regla para que esto \\
{$[373]$} & salga bien. Se trata de encontrar algún patrón. Tiene ese \\
{$[374]$} & decimal, le da esa fracción; tiene ese, le da esa fracción; tiene este, \\
{$[375]$} & le da esa fracción. Hay algo que se comparten entre las fracciones. \\
\hline
\end{tabular}
Ilustración 7. Extracto de transcripción correspondientes al episodio [2.2]. 
Lo mismo ocurre cuando Ana trata la transformación de decimales periódicos y semiperiódicos a fracción, sin embargo en este caso, propone ejemplos resueltos:
[454] Ana: Mire lo que tiene ahí en la pizarra $\left(0, \overline{3}=\frac{3}{9} ; 2, \overline{3}=\frac{21}{9} \ldots\right)$ Son
[455] todos números periódicos, y yo le asocié ya la fracción.
[456] Entonces, ahora vamos a buscar la regla, viendo qué es lo
[457] que está ocurriendo ahí. Mire bien, primero, mire los números,
[458] mire todo y ahí usted podría sacar algunas conclusiones, yo
[459] las voy anotando, y después armamos la regla. No le puedo
[460] decir, no le puedo decir por qué están estos números, pero son
[461] esos, las fracciones asociadas al decimal periódico.

Ilustración 8. Extracto de transcripción correspondientes al episodio [2.3], donde el objetivo de la profesora es transformar los decimales periódicos y semiperiódicos a fracciones.

En ambos extractos el conocimiento de Ana sobre estrategias de enseñanza a partir de la búsqueda de patrones (KMT) se sustenta en su conocimiento acerca de una de las formas de proceder en matemáticas, estableciendo regularidades y buscando patrones, que se asocia con su conocimiento de la práctica matemática (KPM).

En otro episodio (asociado al objetivo de introducir los números racionales), se observa el conocimiento de la enseñanza de Ana, cuando la profesora propone varios ejemplos (KMT) al darse cuenta de que los alumnos pueden quedarse con una idea errónea (KFLM) del concepto de decimal semiperiódico si se les ofrece (solo) un ejemplo concreto:

\begin{tabular}{|ll|}
\hline [294] Ana: & Si a usted le dicen: ¿qué es un número decimal semiperiódico? \\
[295] & ¿Alguien podría explicarlo? \\
[296] Alumno: & Después de la coma hay un número y luego la parte periódica. \\
[297] Ana: & Miren, se quedó con mi ejemplo, solamente cree que debe haber un \\
{$[297]$} & cero y un número [su ejemplo es $0,0 \overline{8}$ ] y después viene lo que es el \\
{$[298]$} & periodo, pero no ocurre así, porque también puedo tener lo \\
{$[299]$} & siguiente: $\mathbf{2 , 3 5}$ y recién tengo un periodo. \\
\hline
\end{tabular}

Ilustración 9. Extracto de transcripción del episodio [1.6], donde el objetivo de la profesora es enseñar a transformar una fracción a número decimal.

El conocimiento de Ana asociado a la importancia de los ejemplos (Rowland, 2008) en el aprendizaje de las matemáticas permite tratar de superar las dificultades de sus alumnos asociadas al aprendizaje de los racionales (KFLM) presentando otros ejemplos donde la 
posición y el número de cifras del período sean distintos - lo que se configura como una de sus estrategias de enseñanza.

En otro extracto (Ilustración 10), asociado al objetivo de discutir la densidad de un conjunto y aclarar las respuestas y el conocimiento de los alumnos al respecto, Ana revela aspectos de su KMT vinculados al modo de subsanar el razonamiento erróneo de sus alumnos, en relación con la noción de densidad:

\begin{tabular}{|ll|}
\hline$[355]$ Ana: & $\ldots$ ¿Por qué el conjunto se dice denso? Aquí hay que hacer dos \\
{$[356]$} & aclaraciones, la mayoría de ustedes que mostró el cuaderno escribió \\
{$[357]$} & "porque el conjunto es infinito". El conjunto sí es infinito, pero eso no \\
{$[358]$} & lo cataloga para ser denso que sea infinito, porque $\mathbf{Z}$ es infinito, los \\
{$[359]$} & N son infinitos, $\mathbf{y}$ ninguno de ellos dos son densos. La densidad no \\
{$[360]$} & tiene que ver con la cantidad de elementos de esa forma, sino tiene que \\
{$[361]$} & ver con cuántos elementos hay entre un racional y otro, ahí hay \\
{$[362]$} & infinitos, por eso se puede decir que es denso. Cosa que no ocurre en \\
{$[363]$} & los naturales, porque si tiene el o y el 1, ihay algún otro natural \\
{$[364]$} & entre medio [en medio]? No, entonces no es denso. \\
\hline
\end{tabular}

Ilustración 10. Extracto de transcripción correspondiente al episodio [2.1], donde la profesora tiene por objetivo aclarar respuestas.

Como se puede observar, los alumnos contestan la pregunta asociando la densidad de los números racionales con la infinitud del conjunto. Sustentada en su conocimiento de este tipo de razonamiento erróneo de los alumnos (KFLM), y para solventar esa confusión, Ana, teniendo por base su KoT, presenta algunos contraejemplos de otros conjuntos infinitos (e.g., naturales, enteros) que no son densos en la recta numérica, resaltando la propiedad topológica de los números racionales - asumiendo la importancia de los ejemplos (e.g., Rowland, 2008) en el proceso de enseñanza. De este modo, se evidencia el conocimiento de las potencialidades del uso de ejemplos en la enseñanza, el conocimiento del contenido matemático como objeto de enseñanza.

En el siguiente mapa de conexiones (Figura 3), se presentan las distintas relaciones identificadas en la práctica de Ana en las sesiones dedicadas al tema de los números racionales: 


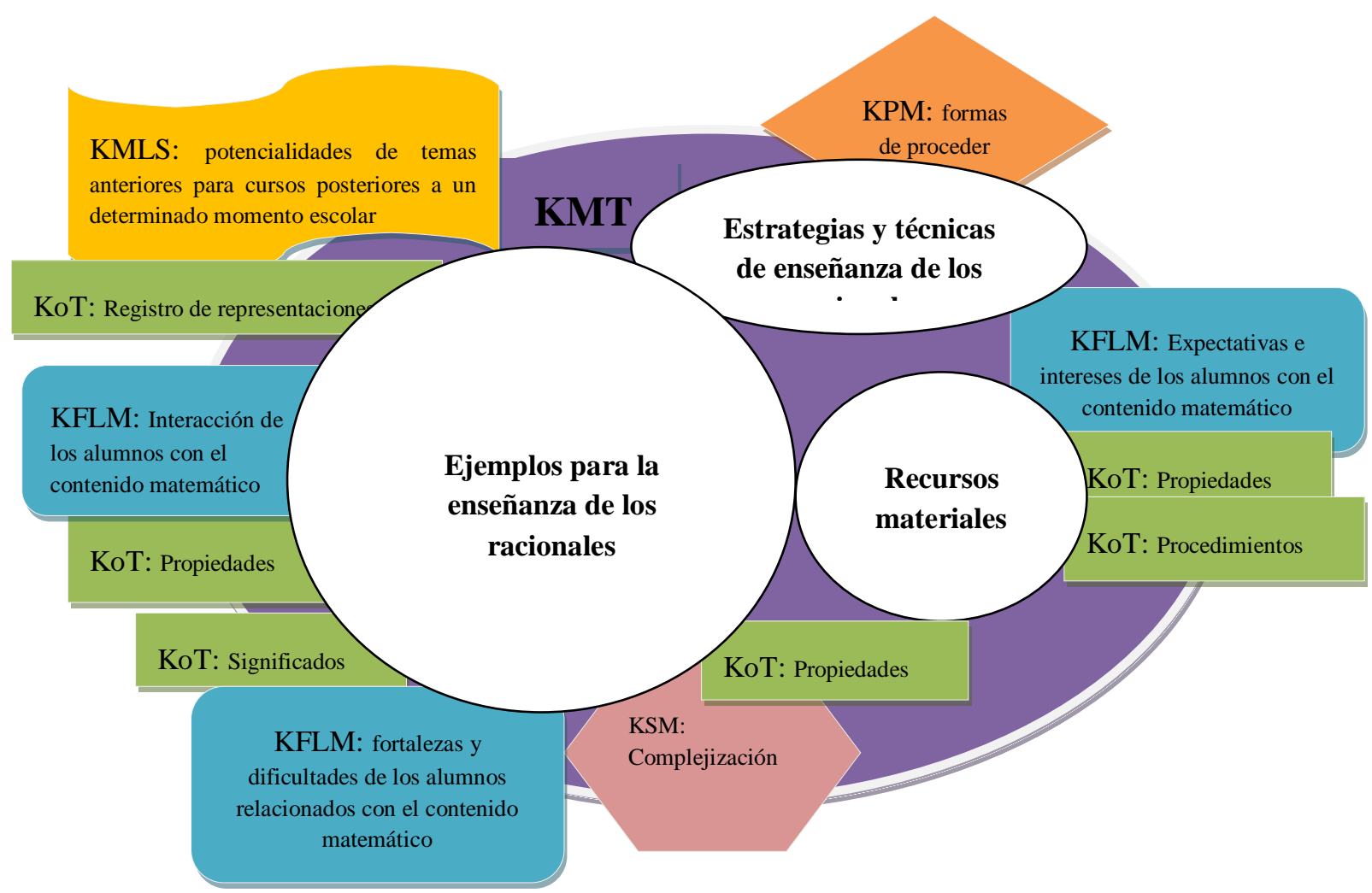

Figura 3. Mapa de conexiones entre el KMT de Ana y otros subdominios del modelo MTSK.

Fuente: Elaboración propia.

La Figura 3 da cuenta de las múltiples y diversas conexiones entre los subdominios del conocimiento especializado de la profesora del estudio, hecho que permite dimensionar la complejidad del constructo del conocimiento del profesor.

Se puede observar que el conocimiento de Ana de la enseñanza de las matemáticas (KMT) está sustentado y/o relacionado, de forma explícita, con los otros cinco subdominios del MTSK. Es notable la comparecencia de las diferentes categorías de los tres subdominios del Conocimiento Matemático (KoT, KSM y KPM), con mayor presencia del KoT, hecho que confirma la estrecha relación entre el conocimiento del contenido (que también se considera especializado) y el conocimiento didáctico del contenido (e.g., Ma, 1999).

Asimismo, se observa que Ana ha tomado decisiones respecto a la enseñanza de los números racionales basándose en su conocimiento de potencialidades de temas anteriores para cursos posteriores (KMLS) y de las dificultades de los alumnos, sus interacciones con el contenido matemático y de los intereses de los alumnos al/para aprender matemáticas (categorías del KFLM), evidenciando de esta manera la interdependencia de estos subdominios y el carácter integrado del conocimiento del profesor sobre el proceso de enseñanza y aprendizaje (e.g. Escudero-Avila, 2015). 
Cabe destacar que el uso de ejemplos para la enseñanza de los racionales ha sido un recurso importante y frecuente en la práctica docente de Ana. Se puede ver que Ana ha recurrido a su conocimiento de ejemplos, que involucra relaciones con conocimientos de distinta índole (Figura 3), en cuatro situaciones distintas y con objetivos diferentes, confirmando la utilidad de los ejemplos en el proceso de enseñanza (e.g., Rowland, 2008).

\section{Comentarios finales}

Los resultados de este estudio permiten evidenciar la operacionalización del uso del modelo del MTSK en el análisis de la práctica docente, teniendo como foco el conocimiento del profesor. Por otro lado, permiten completar con mayor precisión el contenido de cada uno de los subdominios involucrados en el sentido de obtener un mapa amplio de conocimientos y sus relaciones, (necesarios y suficientes) en la práctica del profesor.

La diversidad y complejidad del contenido del conocimiento del profesor son características que hacen difícil la discusión sobre el conocimiento "más adecuado" del docente. Esta dificultad se sustenta no solo en la complejidad de la práctica sino también, por ejemplo, en la multiplicidad de variables (e.g., nivel de escolaridad, diferentes tópicos matemáticos) y en la influencia del contexto cultural en el cual la práctica ocurre (pese a que muchos de los aspectos centrales sean comunes). Asumir tal complejidad incrementa el desafío de determinar el contenido del conocimiento del profesor (e.g., Potari et al., 2013), sin embargo una búsqueda de los elementos caracterizadores de dicho conocimiento puede ayudar en su comprensión y a conceptualizar las formas de promover su desarrollo (Jakobsen et al., 2014).

Las relaciones entre el KMT y los demás subdominios del MTSK nos permiten identificar un conjunto de elementos nucleares del conocimiento del profesor revelado en la práctica, tanto en términos de las influencias del contenido de cada uno de los subdominios (KMT como forma de exteriorizar el conocimiento en la práctica), bien en términos de cuestionar el futuro foco de la formación de modo que posibilite enriquecer el contenido del conocimiento del profesor (en términos de los subdominios), bien crear, ampliar y fortalecer las relaciones entre los distintos subdominios. Se torna así evidente la importancia del conocimiento matemático (KoT, KPM, KSM) y didáctico del contenido (KFLM y KMLS) en la selección y uso de un conjunto diversificado de técnicas de enseñanza (KMT) cuando se pretende explorar tareas con intencionalidad matemática y asociadas a objetivos que permitan que los alumnos sepan lo que hacen y por qué lo hacen - aunque en algunos casos, esos objetivos no se alcanzan completamente debido a posibles lagunas en el propio conocimiento del profesor, en el caso de los racionales.

Estas evidencias ponen de relieve la importancia del conocimiento del profesor en, para y sobre la práctica y nos llevan a problematizar la formación (matemática y didáctica) 
del profesor y los objetivos de esa formación. En este sentido, el mapa de conexiones, presentado en la Figura 3, ilustra algunas de las relaciones entre los distintos subdominios del conocimiento del profesor (son necesarios más estudios al respecto que permitan enriquecer/ampliar el conocimiento de dichas relaciones). Por otra parte, evidenciar estas relaciones puede considerarse como un buen punto de partida para diseñar cursos de formación de profesores que tengan por objetivo el desarrollo del conocimiento del profesor, atendiendo las especificidades de dicho conocimiento - tanto en términos del conocimiento del contenido como didáctico del contenido.

Un futuro desafío es considerar de forma integrada los conocimientos del profesor (e.g., movilizados en la práctica, expresados en cuestionarios, evaluados en ítems) con las acciones nucleares del proceso de enseñanza, tales como atribuir sentido a las respuestas/comentarios de los alumnos proporcionando una retroalimentación constructiva (Jakobsen et al., 2014), presentar las ideas matemáticas, responder a los porqués o hacer preguntas matemáticas productivas (Ball et al., 2008). En esta línea, se hace esencial la preparación de situaciones basadas en la práctica real - recurriendo a una variedad de formas de presentación, tales como videos de episodios, viñetas, respuestas de alumnos o cartoons que evidencien abordajes matemáticamente desafiantes y que sean necesaria y didácticamente significativas.

Tal como muestran las relaciones obtenidas a partir del análisis de la práctica, para que la clase se enfoque en alcanzar objetivos matemáticos es necesario articular de manera significativa la dimensión del conocimiento del contenido (matemática) y la dimensión relacionada con la operacionalización (parte del conocimiento pedagógico del contenido).

Las evidencias del conocimiento del profesor en la práctica, de las relaciones e influencias entre los distintos subdominios del conocimiento, dan indicio de la necesidad de recurrir a un camino (entre muchos) relacionado con los propios objetivos (expresados y alcanzados) de los programas de formación de profesores - e.g., qué conocimientos se pretende desarrollar y cuál es el "perfil" del futuro profesor al terminar su formación. Pensando en nuestra propia práctica como formadores de profesores, los resultados de las investigaciones que estamos realizando, permiten enfocar nuestro trabajo en la búsqueda de una comprensión más amplia del contenido de nuestro propio conocimiento (problematizándolo) y del conocimiento del profesor. Con la intención de ampliar dicho conocimiento, es crucial la conceptualización de tareas para la formación (inicial y continua) que permitan desarrollar ese conocimiento y enfocar la formación donde ésta es más necesaria (Ribeiro \& Carrillo, 2011). 


\section{Referencias}

Ball, D. L., Thames, M. H. \& Phelps, G. (2008). Content knowledge for teaching: what makes it special? Journal of Teacher Education, 59(5), 389-407.

Behr, M.J., Lesh, R., Post, T.R., \& Silver, E.A. (1983). Rational number concepts. In R. Lesh \& M. Landau (Eds.), Acquisition of mathematics concepts and process (pp. 91-126). New York: Academic Press, Inc.

Carrillo, J., Climent, N., Contreras, L. C. \& Muñoz-Catalán, M. C. (2013). Determining Specialized Knowledge for Mathematics Teaching. In B. Ubuz, C. Haser, \& M. A. Mariotti (Eds.), Proceedings VIII Congress of the European Society for Research in Mathematics Education (CERME 8) (pp. 2985-2994). Antalya: Middle East Technical University, Ankara.

Cohen L. \& Manion L. (2002). Métodos de investigación educativa. Madrid: La Muralla.

Escudero-Avila, D. (2015). Una caracterización del conocimiento didáctico del contenido como parte del conocimiento especializado del profesor de matemáticas de secundaria. Tesis doctoral. Huelva: Universidad de Huelva.

Flores, A. (2002). Profound understanding of division of fraction. In B. Litwiller \& G. Bright (Eds.), Making sense of fractions, ratios, and proportions: 2002 Yearbook (pp. 237-246). Reston: NCTM.

Flores-Medrano, E., Escudero-Avila, D., Montes, M., Aguilar, A. \& Carrillo, J. (2014). ¿Cómo se relaciona el conocimiento que tiene el profesor acerca del aprendizaje de las matemáticas con su entendimiento sobre los Espacios de Trabajo Matemático? En I. Gómez-Chacón, J. Escribano, A. Kuzniak \& P.R. Richard (Eds.), Proceedings Fourth ETM Symposium (pp. 473-485), Madrid, España.

Graeber, A., Tirosh, D., \& Glover, R. (1989). Preservice teachers' misconceptions in solving verbal problems in multiplication and division. Journal for Research in Mathematics Education, 20(1), 95-102.

Harel, G., Behr, M., Post, T., \& Lesh, R. (1994). The impact of number type on the solution of multiplication and division problems: Further considerations. In G. Harel \& J. Confrey (Eds.), The development of multiplicative reasoning in the learning of mathematics (pp. 365-388). Albany, NY: SUNY Press.

Jakobsen, A., Ribeiro, C. M. \& Mellone, M. (2014). Norwegian prospective teachers' MKT when interpreting pupils' productions on a fraction task. Nordic Studies in Mathematics Education, 19(3-4), 135-150.

Kieren, T. (1976). On the mathematical, cognitive, and instructional foundations of rational numbers. In R. Lesh (Ed.), Number and measurement: papers from a research workshop (pp. 101-144). Columbus, OH: ERIC/SMEAC.

Kuzniak, A. (2011). L'espace de Travail Mathématique et ses genèses. Annales de didactique et de sciences cognitives. 16, 9-24. 
Lamon, S. (2007). Rational numbers and proportional reasoning. In F. Lester (Ed.), Second handbook of mathematics teaching and learning (pp. 629-667). Greenwich, CT: Information Age Publishing.

Ma, L. (1999). Knowing and teaching elementary mathematics: teachers' understanding of fundamental mathematics in China and the United States. Mahwah, NJ: Erlbaum.

Ministerio de Educación de Chile (2009). Objetivos Fundamentales y Contenidos Mínimos Obligatorios de la Educación Básica y Media. Santiago de Chile: Autor.

Muñoz-Catalán, M.C., Contreras, Luis C., Carrillo, J., Rojas, N., Montes, M.Á. \& Climent, N. (2015). Conocimiento especializado del profesor de matemáticas (MTSK): un modelo analítico para el estudio del conocimiento del profesor de matemáticas. La Gaceta de la Real Sociedad Matemática Española, 18(3), 589-605.

Nye, B., Konstantopoulos, S. \& Hedges, L. V. (2004). How large are teacher effects? Educational Evaluation and Policy Analysis, 26(3), 237-257.

Pinto, H. \& Ribeiro, C. M. (2013). Conhecimento e formação de futuros professores dos primeiros anos - o sentido de número racional. Da Investigação às Práticas, 3(1), 85105.

Potari, D., Berg, C., Charalambous, C., Figueiras, L., Hošpesová, A., Ribeiro, C.M., Santos, L., Skott, J. \& Zehetmeier, S. (2013). Group 17 - From a study of teaching practices to issues in teacher education: Introduction. In B. Ubuz, Ç. Haser \& M. A. Mariotti (Eds.), Atas do CERME 8 (pp. 2896-2907). Antalia, Turquia: ERME.

Ribeiro, C.M. \& Carrillo, J. (2011). The role of beliefs and knowledge in practice. In B. Roesken, \& M. Casper (Eds.), Current state of research on mathematical beliefs XVII MAVI 17 (pp. 192-201). Bochum: Professional School of Education, Ruhr-Universität Bochum.

Ribeiro, C.M., Carrillo, J. \& Monteiro, R. (2012). Cognições e tipo de comunicação do professor de matemática. Exemplificação de um modelo de análise num episódio dividido. Revista Latinoamericana de Investigación en Matemática Educativa, 15(1), 277-310.

Rowland, T. (2008). The purpose, design and use of examples in the teaching of elementary mathematics. Educational Studies in Mathematics, 69, 149-163.

Shulman, L. (1986). Those who understand: Knowledge growth in teaching. Educational Researcher, 15 (2), 4-14.

Stake, R.E. (2007). Investigación con estudio de casos. Madrid: Morata.

Stein, M.K., Smith, M.S., Henningsen, M.A. \& Silver, E.A. (2000). Implementing standardsbased mathematics instruction: a Casebook for Professional Development. New York: Teachers College Press.

Vanhille, L.S., \& Baroody, A.J. (2002). Fraction instruction that fosters multiplicative reasoning. In B. Litwiller (Ed.), Making sense of fractions, ratios, and proportions: 
DOI: http://dx.doi.org/10.20396/zet.v24i3.8648095

NCTM 2002 Yearbook (pp. 224-236). Reston,VA: National Council of Teachers of Mathematics.

Vergnaud, G. (1983). Multiplicative structures. In R. Lesh \& M. Landau (Eds.), Acquisition of mathematics concepts and processes (pp. 127-174). New York, NY: Academic Press.

Zakaryan, D., Ribeiro, C.M., \& Carrillo, J. (sometido). Conocimiento del profesor de los números racionales como objeto de aprendizaje: Un estudio de caso. Perfiles Educativos. 